\title{
A New Inaccuracy Measure for Fuzzy Sets and its Application in Multi-Criteria Decision Making
}

\author{
Rajkumar Verma \\ Department of Mathematics, Jaypee Institute of Information Technology (Deemed University), Noida (U.P.), India \\ E-mail: rkver83@gmail.com \\ Bhu Dev Sharma \\ Department of Mathematics, Jaypee Institute of Information Technology (Deemed University), Noida (U.P.), India \\ E-mail: bhudev.sharma@jit.ac.in, bhudev.sharma@gmail.com
}

\begin{abstract}
In the present paper, a new fuzzy inaccuracy measure is proposed to measure the inaccuracy of a fuzzy set with respect to another fuzzy set. This measure is a modified version of fuzzy inaccuracy proposed in our earlier work. The measure is demonstrated to satisfy some interesting properties, which prepare ground for applications in multi-criteria decision making problems. A method to solve multi-criteria decision making problems with the help of new measure is developed. Finally, a numerical example is given to illustrate the proposed method to solve mu lti-criteria decision-making problem under fuzzy environment.
\end{abstract}

Index Terms - Fuzzy Sets, Fuzzy Entropy, Fuzzy Inaccuracy Measure, Fuzzy Divergence Measure

\section{Introduction}

The concept of fuzzy sets proposed by Zadeh [1] in 1965 has gained wide applications in many areas of science and technology e.g. clustering, image processing, decision making etc. because of its capability to model non-statistical imp recision or vague concepts. Fuzziness, a feature of uncertainty, results from the lack of sharp distinction of being or not being a member of the set. The first attempt to quantify the fuzziness was made in 1968 by Zadeh [2], who introduced a probabilistic framework and defined the entropy of a fuzzy event as weighted Shannon entropy [3]. In 1972, De Luca and Termini [4] formulated axioms with which the fuzzy entropy measure should comply and defined a kind of entropy of a fuzzy setbased on Shannon's function.

In 1992, Bhandari and Pal [5] proposed a measure of fuzzy divergence between two fuzzy sets corresponding to Kullback-Leibler's [6 and 7] measure of divergence. Afterwards, by using the idea of Lin [8], Shang and Jiang [9] introduced a modified version of fuzzy divergence measure proposed in [5].

In 1961, Karridge [10] firstly introduced the idea of inaccuracy measure in information theory as a generalization of Shannon's entropy. It is normal for an observed (say) distribution to differ from a theoretical distribution of a random variable. Kerridge addressed to the problem of defining an (information theoretic) measure of inaccuracy of the distribution to the standard distribution under reference. According to Kerridge [10], the inaccuracy can be regarded as a measure of quantity of missing information. This has been the probabilistic approach. For uncertain phenomena, to cover nonprobabilistic cases, Verma and Sharma [11] defined a measure of inaccuracy in the setting of fuzzy sets theory and studied its applications in multi-criteria decision making under fuzzy environment. There may be rather difficult or extreme cases in which our inaccuracy measure may not applied. In the present communication, to overcome this problem a new fuzzy inaccuracy measure of a fuzzy set with respect to another fuzzy set is proposed. This paper is organized as follows:

In Section 2 some basic definitions related to fuzzy set theory are presented. In Section 3 we introduce a new fuzzy inaccuracy measure of a fuzzy set with respect to another fuzzy set. In Section 4 we study some properties of the proposed fuzzy inaccuracy measure. A weighted fuzzy inaccuracy measure is also defined. In Section 5 a method to solve a multi-criteria decision making problem with the help of weighted fuzzy inaccuracy measure is discussed. Finally, a numerical example is presented to illustrate the procedure of proposed method to solve multi-criteria decision-making and our conclusions are presented in Section 6.

\section{Preliminiries}

In this section, we present some basic concepts related to fuzzy set theory which will be needed in the following analysis.

Definition 1. Fuzzy Set [1]: A fuzzy set $A$ defined in a finite universe of discourse $X=\left\{x_{1}, x_{2}, \ldots, x_{n}\right\}$ is mathematically represented as

$$
A=\left\{\left\langle x, \mu_{A}(x)\right\rangle \mid x \in X\right\},
$$


where $\mu_{A}(x): X \rightarrow[0,1]$ is measure of belongingness or degree of membership of an element $x \in X$ in $A$.

Definition 2. Set Operations on FSs [1]: Let $F S(X)$ denote the family of all FSs in the universe $X$, and let $A, B \in F S(X)$ be two FSs, given by,

$$
\begin{gathered}
A=\left\{\left\langle x, \mu_{A}(x)\right\rangle \mid x \in X\right\}, \\
B=\left\{\left\langle x, \mu_{B}(x)\right\rangle \mid x \in X\right\},
\end{gathered}
$$

then following set operations are defined on FSs:

(i) $A^{C}=\left\{\left\langle x, 1-\mu_{A}(x)\right\rangle \mid x \in X\right\}$;

(ii) $A \cap B=\left\{\left\langle x, \mu_{A}(x) \vee \mu_{B}(x)\right\rangle \mid x \in X\right\}$;

(iii) $A \cup B=\left\{\left\langle x, \mu_{A}(x) \wedge \mu_{B}(x)\right\rangle \mid x \in X\right\}$;

where $\vee, \wedge$ stand for max. and $\min$. operators, respectively.

De Luca and Termini [4] defined fuzzy entropy for a fuzzy set $A$ corresponding to Shannon [3] entropy as

$$
H(A)=-\frac{1}{n} \sum_{i=1}^{n}\left[\begin{array}{c}
\mu_{A}\left(x_{i}\right) \log \mu_{A}\left(x_{i}\right) \\
+\left(1-\mu_{A}\left(x_{i}\right)\right) \log \left(1-\mu_{A}\left(x_{i}\right)\right)
\end{array}\right] .
$$

Corresponding to Kullback-Leibler's [6 and 7] measure of divergence, Bhandari and Pal [5] proposed a fuzzy divergence measure between $A$ and $B$ given by

$$
D(A \mid B)=\frac{1}{n} \sum_{i=1}^{n}\left[\begin{array}{l}
\mu_{A}\left(x_{i}\right) \log \frac{\mu_{A}\left(x_{i}\right)}{\mu_{B}\left(x_{i}\right)} \\
+\left(1-\mu_{A}\left(x_{i}\right)\right) \log \frac{\left(1-\mu_{A}\left(x_{i}\right)\right)}{\left(1-\mu_{B}\left(x_{i}\right)\right)}
\end{array}\right] .
$$

Later, Shang and Jiang [9] pointed out that (3) has a drawback, i.e., when $\mu_{B}\left(x_{i}\right)$ approaches 0 or 1 , its value tends toward infinity. Therefore, they proposed a modified version of fuzzy divergence measure (3), given as

$$
J(A \mid B)=\sum_{i=1}^{n}\left[\begin{array}{l}
\mu_{A}\left(x_{i}\right) \log \frac{\mu_{A}\left(x_{i}\right)}{\frac{\mu_{A}\left(x_{i}\right)+\mu_{B}\left(x_{i}\right)}{2}} \\
+\left(1-\mu_{A}\left(x_{i}\right)\right) \log \frac{\left(1-\mu_{A}\left(x_{i}\right)\right)}{1-\left(\frac{\mu_{A}\left(x_{i}\right)+\mu_{B}\left(x_{i}\right)}{2}\right)}
\end{array}\right] .
$$

Recently, Verma and Sharma [11] defined a measure of inaccuracy of fuzzy set $B$ with respect to fuzzy set $A$, corresponding to Karridge [10] inaccuracy measure as

$$
I(A ; B)=-\frac{1}{n} \sum_{i=1}^{n}\left[\begin{array}{c}
\mu_{A}\left(x_{i}\right) \log \mu_{B}\left(x_{i}\right) \\
+\left(1-\mu_{A}\left(x_{i}\right)\right) \log \left(1-\mu_{B}\left(x_{i}\right)\right)
\end{array}\right] .
$$

It may be noted that (5) has some problems. If $\mu_{A}\left(x_{i}\right) \neq 0$ and $\mu_{B}\left(x_{i}\right)=0$ or $\mu_{A}\left(x_{i}\right) \neq 1$ and $\mu_{B}\left(x_{i}\right)=1$, for any $x_{i}$, then $I(A ; B)$ becomes infinite.

For example: Let $A=\{\langle x, 0.4\rangle\}, B=\left\{\left\langle x_{1}, 0.0\right\rangle\right\}$ and $C=\left\{\left\langle x_{1}, 1\right\rangle\right\}$ be three fuzzy sets, then

$$
I(A ; B)=\infty \text { and } I(A ; C)=\infty .
$$

To overcome above mentioned drawback of fuzzy inaccuracy, in the next section, we propose a new fuzzy inaccuracy measure of a fuzzy set with respect to anotherfuzzy set.

\section{A New Fuzzy Inaccuracy Measure}

We proceed with following formal definition:

Definition 3: Let $A$ and $B$ be two fuzzy sets defined 1n a finite universe of discourse $X=\left\{x_{1}, x_{2}, \ldots, x_{n}\right\}$ having the membership values $\mu_{A}\left(x_{i}\right), i=1,2, \ldots, n$ and $\mu_{B}\left(x_{i}\right), i=1,2, \ldots, n$ respectively.

We define the measure of inaccuracy of fuzzy set $B$ with respect to fuzzy set $A$, as

$$
K(A ; B)=-\frac{1}{n} \sum_{i=1}^{n}\left[\begin{array}{l}
\mu_{A}\left(x_{i}\right) \log \left(\frac{\mu_{A}\left(x_{i}\right)+\mu_{B}\left(x_{i}\right)}{2}\right) \\
+\left(1-\mu_{A}\left(x_{i}\right)\right) \log \left(\frac{2-\mu_{A}\left(x_{i}\right)-\mu_{B}\left(x_{i}\right)}{2}\right)
\end{array}\right]
$$

The $K(A ; B)$, is well defined and is independent of the values of $\mu_{A}\left(x_{i}\right)$ and $\mu_{B}\left(x_{i}\right)$. From both the definitions of $I(A ; B)$ and $K(A ; B)$, it is easy to see that $K(A ; B)$ can be described in the terms of $I(A ; B)$ as follows

$$
K(A ; B)=I\left(A ; \frac{A+B}{2}\right)
$$

It is also interesting to note that when $A=B$, then (6) reduces to (2), the measure of fuzzy entropy given by De Luca and Termini in [4].

Now, again consider above example with measure (6), we get

$$
K(A ; B)=1.0627 \text { and } K(A ; C)=0.9726 .
$$

The good part is that the new measure shares properties with measure (5).

\section{Properties of Fuzzy Inaccuracy Measure $K(A ; B)$}

The measure of fuzzy inaccuracy defined in (6) has the following properties: 
Theorem 1: For $A, B \in F S(X)$,

$$
K(A ; B) \leq \frac{1}{2}[I(A ; B)+H(A)] .
$$

Proof: Since $\mu_{A}\left(x_{i}\right) \geq 0$ and $\mu_{B}\left(x_{i}\right) \geq 0$ for any $x_{i} \in X$, by the inequality of the arithmetic and geometric mean, we have

$$
\frac{\mu_{A}\left(x_{i}\right)+\mu_{B}\left(x_{i}\right)}{2} \geq \sqrt{\mu_{A}\left(x_{i}\right) \mu_{B}\left(x_{i}\right)},
$$

for any $x_{i} \in X$

and

$$
\frac{2-\mu_{A}\left(x_{i}\right)-\mu_{B}\left(x_{i}\right)}{2} \geq \sqrt{\left(1-\mu_{A}\left(x_{i}\right)\right)\left(1-\mu_{B}\left(x_{i}\right)\right)},
$$

for any $x_{i} \in X$

Thus,

$$
\begin{aligned}
& K(A ; B) \\
& =-\frac{1}{n} \sum_{i=1}^{n}\left[\begin{array}{l}
\mu_{A}\left(x_{i}\right) \log \left(\frac{\mu_{A}\left(x_{i}\right)+\mu_{B}\left(x_{i}\right)}{2}\right) \\
+\left(1-\mu_{A}\left(x_{i}\right)\right) \log \left(\frac{2-\mu_{A}\left(x_{i}\right)-\mu_{B}\left(x_{i}\right)}{2}\right)
\end{array}\right] \\
& \leq-\frac{1}{n} \sum_{i=1}^{n}\left[\begin{array}{l}
\mu_{A}\left(x_{i}\right) \log \left(\sqrt{\mu_{A}\left(x_{i}\right) \mu_{B}\left(x_{i}\right)}\right) \\
+\left(1-\mu_{A}\left(x_{i}\right)\right) \log \left(\sqrt{\left(1-\mu_{A}\left(x_{i}\right)\right)\left(1-\mu_{B}\left(x_{i}\right)\right)}\right)
\end{array}\right] \\
& =-\frac{1}{2 n} \sum_{i=1}^{n}\left[\begin{array}{l}
\mu_{A}\left(x_{i}\right) \log \left(\mu_{A}\left(x_{i}\right) \mu_{B}\left(x_{i}\right)\right) \\
+\left(1-\mu_{A}\left(x_{i}\right)\right) \log \left(\left(1-\mu_{A}\left(x_{i}\right)\right)\left(1-\mu_{B}\left(x_{i}\right)\right)\right)
\end{array}\right] \\
& =-\frac{1}{2 n} \sum_{i=1}^{n}\left[\begin{array}{l}
\mu_{A}\left(x_{i}\right)\left(\log \left(\mu_{A}\left(x_{i}\right)\right)+\log \left(\mu_{B}\left(x_{i}\right)\right)\right) \\
+\left(1-\mu_{A}\left(x_{i}\right)\right)\left(\begin{array}{l}
\log \left(1-\mu_{A}\left(x_{i}\right)\right) \\
+\log \left(1-\mu_{B}\left(x_{i}\right)\right)
\end{array}\right)
\end{array}\right] \\
& =\frac{1}{2}[I(A ; B)+H(A)] .
\end{aligned}
$$

This proves the theorem.

Theorem 2: $K(A ; B)=0$ if and only if either $\quad \mu_{A}\left(x_{i}\right)=\mu_{B}\left(x_{i}\right)=0 \quad$ or $\quad \mu_{A}\left(x_{i}\right)=\mu_{B}\left(x_{i}\right)=1$ $\forall i=1,2, \ldots, n$.

Proof: First let $K(A ; B)=0$, then

$$
-\frac{1}{n} \sum_{i=1}^{n}\left[\begin{array}{l}
\log \left(\frac{\mu_{A}\left(x_{i}\right)+\mu_{B}\left(x_{i}\right)}{2}\right) \\
+\left(1-\mu_{A}\left(x_{i}\right)\right) \log \left(\frac{2-\mu_{A}\left(x_{i}\right)-\mu_{B}\left(x_{i}\right)}{2}\right)
\end{array}\right]=0
$$

$$
\left[\begin{array}{l}
\mu_{A}\left(x_{i}\right) \log \left(\frac{\mu_{A}\left(x_{i}\right)+\mu_{B}\left(x_{i}\right)}{2}\right) \\
+\left(1-\mu_{A}\left(x_{i}\right)\right) \log \left(\frac{2-\mu_{A}\left(x_{i}\right)-\mu_{B}\left(x_{i}\right)}{2}\right)
\end{array}\right]=0, \quad \forall i .
$$

The above relation holds only when either $\mu_{A}\left(x_{i}\right)=\mu_{B}\left(x_{i}\right)=0$ or $\mu_{A}\left(x_{i}\right)=\mu_{B}\left(x_{i}\right)=1$ for all $\forall i$.

Conversely, let either $\mu_{A}\left(x_{i}\right)=\mu_{B}\left(x_{i}\right)=0$ or $\mu_{A}\left(x_{i}\right)=\mu_{B}\left(x_{i}\right)=1$, this implies

$$
\left[\begin{array}{l}
\mu_{A}\left(x_{i}\right) \log \left(\frac{\mu_{A}\left(x_{i}\right)+\mu_{B}\left(x_{i}\right)}{2}\right) \\
+\left(1-\mu_{A}\left(x_{i}\right)\right) \log \left(\frac{2-\mu_{A}\left(x_{i}\right)-\mu_{B}\left(x_{i}\right)}{2}\right)
\end{array}\right]=0, \forall i
$$

or

$$
K(A ; B)=0 .
$$

This proves the theorem.

To prove results given in Theorems ahead, we will consider separation of $X$ into two parts $X_{1}$ and $X_{2}$, such that

$$
\begin{aligned}
& X_{1}=\left\{x \mid x \in X, \mu_{A}(x) \geq \mu_{B}\left(\mathrm{x}_{\mathrm{i}}\right)\right\}, \\
& X_{2}=\left\{x \mid x \in X, \mu_{B}(x)<\mu_{A}\left(x_{i}\right)\right\} .
\end{aligned}
$$

Theorem 3: For $A, B, C \in F S(X)$,

(i) $K(A \cup B ; C) \leq K(A ; C)+K(B ; C)$;

(ii) $K(A \cap B ; C) \leq K(A ; C)+K(B ; C)$.

Proof: In the following, we prove only (i), proof of (ii) can be formulated analogously.

(i) Let us consider the following expression

$$
\begin{aligned}
& K(A ; C)+K(B ; C)-K(A \cup B ; C) \\
& =-\frac{1}{n} \sum_{i=1}^{n}\left[\begin{array}{l}
\mu_{A}\left(x_{i}\right) \log \left(\frac{\mu_{A}\left(x_{i}\right)+\mu_{C}\left(x_{i}\right)}{2}\right) \\
+\left(1-\mu_{A}\left(x_{i}\right)\right) \log \left(\frac{2-\mu_{A}\left(x_{i}\right)-\mu_{C}\left(x_{i}\right)}{2}\right)
\end{array}\right] \\
& -\frac{1}{n} \sum_{i=1}^{n}\left[\begin{array}{l}
\mu_{B}\left(x_{i}\right) \log \left(\frac{\mu_{B}\left(x_{i}\right)+\mu_{C}\left(x_{i}\right)}{2}\right) \\
+\left(1-\mu_{B}\left(x_{i}\right)\right) \log \left(\frac{2-\mu_{B}\left(x_{i}\right)-\mu_{C}\left(x_{i}\right)}{2}\right)
\end{array}\right] \\
& +\frac{1}{n} \sum_{i=1}^{n}\left[\begin{array}{c}
\left(\mu_{A}\left(x_{i}\right) \vee \mu_{B}\left(x_{i}\right)\right) \log \frac{\left(\begin{array}{c}
\left(\mu_{A}\left(x_{i}\right) \vee \mu_{B}\left(x_{i}\right)\right) \\
+\mu_{C}\left(x_{i}\right)
\end{array}\right)}{2} \\
+\left(1-\left(\mu_{A}\left(x_{i}\right) \vee \mu_{B}\left(x_{i}\right)\right)\right) \log \left(\frac{\left.2-\left(\mu_{A}\left(x_{i}\right) \vee \mu_{B}\left(x_{i}\right)\right)\right)}{2}-\mu_{C}\left(x_{i}\right)\right. \\
2
\end{array}\right]
\end{aligned}
$$

I.J. Intelligent Systems and Applications, 2014, 05, 62-69 
$=-\frac{1}{n}\left[\begin{array}{c}\sum_{x_{i} \in X_{2}}\left(\begin{array}{l}\mu_{A}\left(x_{i}\right) \log \left(\frac{\mu_{A}\left(x_{i}\right)+\mu_{C}\left(x_{i}\right)}{2}\right) \\ +\left(1-\mu_{A}\left(x_{i}\right)\right) \log \left(\frac{2-\mu_{A}\left(x_{i}\right)-\mu_{C}\left(x_{i}\right)}{2}\right)\end{array}\right) \\ +\sum_{x_{i} \in X_{1}}\left(\begin{array}{l}\mu_{B}\left(x_{i}\right) \log \left(\frac{\mu_{B}\left(x_{i}\right)+\mu_{C}\left(x_{i}\right)}{2}\right) \\ +\left(1-\mu_{B}\left(x_{i}\right)\right) \log \left(\frac{2-\mu_{B}\left(x_{i}\right)-\mu_{C}\left(x_{i}\right)}{2}\right)\end{array}\right)\end{array}\right]$ $\geq 0$

This proves the theorem.

Theorem 4: For $A, B, C \in F S(X)$,

$$
K(A \cup B ; C)+K(A \cap B ; C)=K(A ; B)+K(A ; C)
$$

Proof: From definition in (6), we have

$$
\begin{gathered}
K(A \cup B ; C) \\
=-\frac{1}{n} \sum_{i=1}^{n}\left[\begin{array}{c}
\left(\mu_{A}\left(x_{i}\right) \vee \mu_{B}\left(x_{i}\right)\right) \log \left(\frac{\left(\mu_{A}\left(x_{i}\right) \vee \mu_{B}\left(x_{i}\right)\right)+\mu_{C}\left(x_{i}\right)}{2}\right) \\
+\left(1-\left(\mu_{A}\left(x_{i}\right) \vee \mu_{B}\left(x_{i}\right)\right)\right) \log \left(\frac{2-\left(\mu_{A}\left(x_{i}\right) \vee \mu_{B}\left(x_{i}\right)\right)}{2}\right)
\end{array}\right] \\
=-\frac{1}{n}\left[\begin{array}{c}
\sum_{x_{i} \in X_{1}}\left(\begin{array}{c}
\left.\mu_{A}\right) \\
+\left(x_{i}\right) \log \left(\frac{\mu_{A}\left(x_{i}\right)+\mu_{C}\left(x_{i}\right)}{2}\right) \\
+\left(1-\mu_{A}\left(x_{i}\right)\right) \log \left(\frac{2-\mu_{A}\left(x_{i}\right)-\mu_{C}\left(x_{i}\right)}{2}\right)
\end{array}\right] . \\
\left.+\sum_{x_{i} \in X_{2}}\left(\begin{array}{c}
\mu_{B}\left(x_{i}\right) \log \left(\frac{\mu_{B}\left(x_{i}\right)+\mu_{C}\left(x_{i}\right)}{2}\right) \\
+\left(1-\mu_{B}\left(x_{i}\right)\right) \log \left(\frac{2-\mu_{B}\left(x_{i}\right)-\mu_{C}\left(x_{i}\right)}{2}\right)
\end{array}\right)\right]
\end{array}\right]
\end{gathered}
$$

and

$$
\begin{aligned}
& K(A \cap B ; C) \\
& =-\frac{1}{n} \sum_{i=1}^{n}\left[\begin{array}{c}
\left(\mu_{A}\left(x_{i}\right) \wedge \mu_{B}\left(x_{i}\right)\right) \log \left(\frac{\left(\mu_{A}\left(x_{i}\right) \wedge \mu_{B}\left(x_{i}\right)\right)+\mu_{C}\left(x_{i}\right)}{2}\right) \\
+\left(1-\left(\mu_{A}\left(x_{i}\right) \wedge \mu_{B}\left(x_{i}\right)\right)\right) \log \left(\frac{2-\left(\mu_{A}\left(x_{i}\right) \wedge \mu_{B}\left(x_{i}\right)\right)}{-\mu_{C}\left(x_{i}\right)}\right)
\end{array}\right] \\
& =-\frac{1}{n}\left[\begin{array}{c}
\sum_{x_{i} \in X_{1}}\left(\begin{array}{c}
\mu_{B}\left(x_{i}\right) \log \frac{\mu_{B}\left(x_{i}\right)+\mu_{C}\left(x_{i}\right)}{2} \\
+\left(1-\mu_{B}\left(x_{i}\right)\right) \log \left(\frac{2-\mu_{B}\left(x_{i}\right)-\mu_{C}\left(x_{i}\right)}{2}\right)
\end{array}\right) \\
+\sum_{x_{i} \in X_{2}}\left(\begin{array}{l}
\mu_{A}\left(x_{i}\right) \log \frac{\mu_{A}\left(x_{i}\right)+\mu_{C}\left(x_{i}\right)}{2} \\
+\left(1-\mu_{A}\left(x_{i}\right)\right) \log \left(\frac{2-\mu_{A}\left(x_{i}\right)-\mu_{C}\left(x_{i}\right)}{2}\right)
\end{array}\right)
\end{array}\right] .
\end{aligned}
$$

Adding (15) and (16) we get the result.

Theorem 5: For $A, B \in F S(X)$,

(i) $K(A ; A \cup B)+K(A ; A \cap B)=H(A)+K(A ; B)$;

(ii) $K(B ; A \bigcup B)+K(B ; A \cap B)=H(B)+K(B ; A)$.

Proof: In the following, we prove only (i), proof of (ii) can be formulated analogously.

(i) Using definition in (6), we first have

$K(A ; A \cup B)$

$$
\left.=-\frac{1}{n} \sum_{i=1}^{n}\left[\begin{array}{c}
\mu_{A}\left(x_{i}\right) \log \left(\frac{\mu_{A}\left(x_{i}\right)+\left(\mu_{A}\left(x_{i}\right) \vee \mu_{B}\left(x_{i}\right)\right)}{2}\right) \\
+\left(1-\mu_{A}\left(x_{i}\right)\right) \log \left(\frac{-\left(\mu_{A}\left(x_{i}\right) \vee \mu_{B}\left(x_{i}\right)\right)}{2}\right)
\end{array}\right)\right]
$$

$$
=-\frac{1}{n}\left[\begin{array}{c}
\sum_{x_{i} \in X_{1}}\left(\begin{array}{r}
\mu_{A}\left(x_{i}\right) \log \left(\frac{\mu_{A}\left(x_{i}\right)+\mu_{A}\left(x_{i}\right)}{2}\right) \\
+\left(1-\mu_{A}\left(x_{i}\right)\right) \log \left(\frac{2-\mu_{A}\left(x_{i}\right)}{2}\right) \\
+\sum_{A}\left(x_{i}\right) \\
2
\end{array}\right) \\
\left(\begin{array}{c}
\mu_{A}\left(x_{i}\right) \log \left(\frac{\mu_{A}\left(x_{i}\right)+\mu_{B}\left(x_{i}\right)}{2}\right) \\
2
\end{array}\right) \\
+\left(1-\mu_{A}\left(x_{i}\right)\right) \log \left(\begin{array}{c}
2-\mu_{A}\left(x_{i}\right) \\
-\mu_{B}\left(x_{i}\right) \\
2
\end{array}\right)
\end{array}\right] .
$$

Next, again from definition in (6), we have

$$
K(A ; A \cap B)
$$

$$
\begin{gathered}
\left.=-\frac{1}{n} \sum_{i=1}^{n}\left[\begin{array}{c}
\mu_{A}\left(x_{i}\right) \log \left(\frac{\mu_{A}\left(x_{i}\right)+\left(\mu_{A}\left(x_{i}\right) \wedge \mu_{B}\left(x_{i}\right)\right)}{2}\right) \\
+\left(1-\mu_{A}\left(x_{i}\right)\right) \log \left(\frac{-\left(\mu_{A}\left(x_{i}\right) \wedge \mu_{B}\left(x_{i}\right)\right)}{2}\right)
\end{array}\right)\right] \\
=-\frac{1}{n}\left[\begin{array}{c}
\sum_{x_{i} \in X_{1}}\left(\begin{array}{c}
\mu_{A}\left(x_{i}\right) \log \left(\frac{\mu_{A}\left(x_{i}\right)+\mu_{B}\left(x_{i}\right)}{2}\right) \\
+\left(1-\mu_{A}\left(x_{i}\right)\right) \log \left(\frac{2-\mu_{A}\left(x_{i}\right)-\mu_{B}\left(x_{i}\right)}{2}\right)
\end{array}\right) \\
+\sum_{x_{i} \in X_{2}}\left(\begin{array}{c}
\mu_{A}\left(x_{i}\right) \log \left(\frac{\mu_{A}\left(x_{i}\right)+\mu_{A}\left(x_{i}\right)}{2}\right) \\
+\left(1-\mu_{A}\left(x_{i}\right)\right) \log \left(\frac{2-\mu_{A}\left(x_{i}\right)-\mu_{A}\left(x_{i}\right)}{2}\right)
\end{array}\right)
\end{array}\right] .
\end{gathered}
$$


Adding (17) and (18) we get the result.

Theorem 6: For $A, B \in F S(X)$, then

$$
\begin{aligned}
K(A \bigcup B ; A & \cap B)+K(A \cap B ; A \cup B) \\
= & K(A ; B)+K(B ; A)=2 H\left(\frac{A+B}{2}\right) .
\end{aligned}
$$

Proof: By using definition in (6), we first have

$$
K(A \cup B ; A \cap B)
$$

$$
\begin{aligned}
& =-\frac{1}{n} \sum_{i=1}^{n}\left[\begin{array}{c}
\left(\mu_{A}\left(x_{i}\right) \vee \mu_{B}\left(x_{i}\right)\right) \log \left(\begin{array}{c}
\left(\mu_{A}\left(x_{i}\right) \vee \mu_{B}\left(x_{i}\right)\right) \\
+\left(\mu_{A}\left(x_{i}\right) \wedge \mu_{B}\left(x_{i}\right)\right) \\
2
\end{array}\right) \\
+\left(1-\left(\mu_{A}\left(x_{i}\right) \vee \mu_{B}\left(x_{i}\right)\right)\right) \log \left(\begin{array}{c}
2-\left(\mu_{A}\left(x_{i}\right) \vee \mu_{B}\left(x_{i}\right)\right) \\
-\left(\mu_{A}\left(x_{i}\right) \wedge \mu_{B}\left(x_{i}\right)\right) \\
2
\end{array}\right)
\end{array}\right] \\
& =-\frac{1}{n}\left[\begin{array}{c}
\sum_{x_{i} \in X_{1}}\left(\begin{array}{l}
\mu_{A}\left(x_{i}\right) \log \left(\frac{\mu_{A}\left(x_{i}\right)+\mu_{B}\left(x_{i}\right)}{2}\right) \\
+\left(1-\mu_{A}\left(x_{i}\right)\right) \log \left(\frac{2-\mu_{A}\left(x_{i}\right)-\mu_{B}\left(x_{i}\right)}{2}\right)
\end{array}\right) \\
+\sum_{x_{i} \in X_{2}}\left(\begin{array}{l}
\mu_{B}\left(x_{i}\right) \log \left(\frac{\mu_{B}\left(x_{i}\right)+\mu_{A}\left(x_{i}\right)}{2}\right) \\
+\left(1-\mu_{B}\left(x_{i}\right)\right) \log \left(\frac{2-\mu_{B}\left(x_{i}\right)-\mu_{A}\left(x_{i}\right)}{2}\right)
\end{array}\right)
\end{array}\right] .
\end{aligned}
$$

Next, again from definition in (6), we have

$$
K(A \cap B ; A \cup B)
$$$$
=-\frac{1}{n} \sum_{i=1}^{n}\left[\begin{array}{c}
\left(\mu_{A}\left(x_{i}\right) \wedge \mu_{B}\left(x_{i}\right)\right) \log \left(\begin{array}{l}
\left(\mu_{A}\left(x_{i}\right) \wedge \mu_{B}\left(x_{i}\right)\right) \\
+\left(\mu_{A}\left(x_{i}\right) \vee \mu_{B}\left(x_{i}\right)\right) \\
2
\end{array}\right) \\
+\left(1-\left(\mu_{A}\left(x_{i}\right) \wedge \mu_{B}\left(x_{i}\right)\right)\right) \log \left(\begin{array}{c}
2-\left(\mu_{A}\left(x_{i}\right) \wedge \mu_{B}\left(x_{i}\right)\right) \\
\frac{-\left(\mu_{A}\left(x_{i}\right) \vee \mu_{B}\left(x_{i}\right)\right)}{2}
\end{array}\right)
\end{array}\right]
$$$$
=-\frac{1}{n}\left[\begin{array}{c}
\sum_{x_{i} \in X_{1}}\left(\begin{array}{c}
\mu_{B}\left(x_{i}\right) \log \left(\frac{\mu_{B}\left(x_{i}\right)+\mu_{A}\left(x_{i}\right)}{2}\right) \\
+\left(1-\mu_{B}\left(x_{i}\right)\right) \log \left(\frac{2-\mu_{B}\left(x_{i}\right)-\mu_{A}\left(x_{i}\right)}{2}\right)
\end{array}\right) \\
+\sum_{x_{i} \in X_{2}}\left(\begin{array}{c}
\mu_{A}\left(x_{i}\right) \log \left(\frac{\mu_{A}\left(x_{i}\right)+\mu_{B}\left(x_{i}\right)}{2}\right) \\
+\left(1-\mu_{A}\left(x_{i}\right)\right) \log \left(\frac{2-\mu_{A}\left(x_{i}\right)-\mu_{B}\left(x_{i}\right)}{2}\right)
\end{array}\right)
\end{array}\right] .
$$

Finally, adding (19) and (20) we get the result.

Theorem 7: For $A, B, C \in F S(X)$,

$$
K(A ; B \bigcup C)+K(A ; B \cap C)=K(A ; B)+K(A ; C) .
$$

Proof: Let us separate $X$ into two parts $X_{1}$ and $X_{2}$, such that

$$
\begin{aligned}
& X_{1}=\left\{x \mid x \in X, \mu_{B}(x) \geq \mu_{C}\left(\mathrm{x}_{\mathrm{i}}\right)\right\}, \\
& X_{2}=\left\{x \mid x \in X, \mu_{B}(x)<\mu_{C}\left(x_{i}\right)\right\} .
\end{aligned}
$$

We then have,

$$
K(A ; B \cup C)
$$

$$
=-\frac{1}{n} \sum_{i=1}^{n}\left[\begin{array}{l}
\mu_{A}\left(x_{i}\right) \log \left(\frac{\mu_{A}\left(x_{i}\right)+\left(\mu_{B}\left(x_{i}\right) \vee \mu_{C}\left(x_{i}\right)\right)}{2}\right) \\
+\left(1-\mu_{A}\left(x_{i}\right)\right) \log \left(\frac{2-\mu_{A}\left(x_{i}\right)-\left(\mu_{B}\left(x_{i}\right) \vee \mu_{C}\left(x_{i}\right)\right)}{2}\right)
\end{array}\right]
$$$$
=-\frac{1}{n}\left[\begin{array}{c}
\sum_{x_{i} \in X_{1}}\left(\begin{array}{c}
\mu_{A}\left(x_{i}\right) \log \left(\frac{\mu_{A}\left(x_{i}\right)+\mu_{B}\left(x_{i}\right)}{2}\right) \\
+\left(1-\mu_{A}\left(x_{i}\right)\right) \log \left(\frac{2-\mu_{A}\left(x_{i}\right)-\mu_{B}\left(x_{i}\right)}{2}\right)
\end{array}\right) \\
+\sum_{x_{i} \in X_{2}}\left(\begin{array}{l}
\mu_{A}\left(x_{i}\right) \log \left(\frac{\mu_{A}\left(x_{i}\right)+\mu_{C}\left(x_{i}\right)}{2}\right) \\
+\left(1-\mu_{A}\left(x_{i}\right)\right) \log \left(\frac{2-\mu_{A}\left(x_{i}\right)-\mu_{C}\left(x_{i}\right)}{2}\right)
\end{array}\right)
\end{array}\right]
$$

And

$$
K(A ; B \cap C)
$$

$$
=-\frac{1}{n} \sum_{i=1}^{n}\left[\begin{array}{l}
\mu_{A}\left(x_{i}\right) \log \left(\frac{\mu_{A}\left(x_{i}\right)+\left(\mu_{B}\left(x_{i}\right) \wedge \mu_{C}\left(x_{i}\right)\right)}{2}\right) \\
+\left(1-\mu_{A}\left(x_{i}\right)\right) \log \left(\frac{2-\mu_{A}\left(x_{i}\right)-\left(\mu_{B}\left(x_{i}\right) \wedge \mu_{C}\left(x_{i}\right)\right)}{2}\right)
\end{array}\right]
$$

$$
=-\frac{1}{n}\left[\begin{array}{c}
\sum_{x_{i} \in X_{1}}\left(\begin{array}{c}
\mu_{A}\left(x_{i}\right) \log \left(\frac{\mu_{A}\left(x_{i}\right)+\mu_{C}\left(x_{i}\right)}{2}\right) \\
+\left(1-\mu_{A}\left(x_{i}\right)\right) \log \left(\frac{2-\mu_{A}\left(x_{i}\right)-\mu_{C}\left(x_{i}\right)}{2}\right)
\end{array}\right) \\
+\sum_{x_{i} \in X_{2}}\left(\begin{array}{c}
\mu_{A}\left(x_{i}\right) \log \left(\frac{\mu_{A}\left(x_{i}\right)+\mu_{B}\left(x_{i}\right)}{2}\right) \\
+\left(1-\mu_{A}\left(x_{i}\right)\right) \log \left(\frac{2-\mu_{A}\left(x_{i}\right)-\mu_{B}\left(x_{i}\right)}{2}\right)
\end{array}\right)
\end{array}\right] .
$$

Adding (23) and (24) we obtain,

$$
K(A ; B \bigcup C)+K(A ; B \bigcap C)=K(A ; B)+K(A ; C) .
$$

This proves the theorem.

Corollary : Let $A, B, C \in F S(X)$, then

$$
\begin{aligned}
K(A ; B \cup C)+K(A ; B \cap C) & \\
=K(A \cup B ; C)+ & K(A \cap B ; C) \\
& =K(A ; B)+K(A ; C) .
\end{aligned}
$$


Proof: It obvious follows from Theorems 4 and 7. Theorem 8: For two fuzzy sets $A$ and $B$,

$$
H(A) \leq K(A ; B),
$$

with equality if and only if $A=B$, i.e., $\mu_{A}\left(x_{i}\right)=\mu_{B}\left(x_{i}\right) \forall x_{i} \in X$.

Proof: Let us consider the following expression

$$
K(A ; B)-H(A) .
$$

After putting the value of $K(A ; B)$ and $H(A)$ in (27), we get

$$
\begin{aligned}
& =\frac{1}{n} \sum_{i=1}^{n}\left[\begin{array}{l}
\mu_{A}\left(x_{i}\right) \log \frac{\mu_{A}\left(x_{i}\right)}{\left(\frac{\mu_{A}\left(x_{i}\right)+\mu_{B}\left(x_{i}\right)}{2}\right)} \\
+\left(1-\mu_{A}\left(x_{i}\right)\right) \log \frac{\left(1-\mu_{A}\left(x_{i}\right)\right)}{\left(\frac{2-\mu_{A}\left(x_{i}\right)-\mu_{B}\left(x_{i}\right)}{2}\right)}
\end{array}\right] \\
& =J(A \mid B) .
\end{aligned}
$$

From [8], we know that $J(A \mid B) \geq 0$ with equality if and only if $A=B$, i.e., $\mu_{A}\left(x_{i}\right)=\mu_{B}\left(x_{i}\right) \forall x_{i} \in X$.

Hence

$$
K(A ; B)-H(A) \geq 0,
$$

with equality if and only if $A=B$, i.e., $\mu_{A}\left(x_{i}\right)=\mu_{B}\left(x_{i}\right) \forall x_{i} \in X$.

This proves the theorem.

Considering that the elements in the universe of discourse $X=\left\{x_{1}, x_{2}, \ldots, x_{n}\right\}$ may have different importance, we define the weighted fuzzy inaccuracy measure of fuzzy set $B$ with respect to fuzzy set $A$, as

Definition 4: Given two fuzzy sets $A$ and $B$ defined in a finite universe of discourse $X=\left\{x_{1}, x_{2}, \ldots, x_{n}\right\}$ with membership values $\mu_{A}\left(x_{i}\right)$ and $\mu_{B}\left(x_{i}\right), i=1,2, \ldots, n$, with weight vector $w=\left(w_{1}, w_{2}, \ldots, w_{n}\right)^{T}$ of the elements $x_{i}, i=1,2, \ldots, n$, such that $w_{i} \geq 0$ and $\sum_{i=1}^{n} w_{i}=1$, the weighted fuzzy inaccuracy of fuzzy set $B$ with respect to fuzzy set $A$, is defined as

$$
\begin{aligned}
& K(A ; B, w) \\
& =-\sum_{i=1}^{n} w_{i}\left[\begin{array}{l}
\mu_{A}\left(x_{i}\right) \log \left(\frac{\mu_{A}\left(x_{i}\right)+\mu_{B}\left(x_{i}\right)}{2}\right) \\
\left.+\left(1-\mu_{A}\left(x_{i}\right)\right) \log \left(\frac{2-\mu_{A}\left(x_{i}\right)-\mu_{B}\left(x_{i}\right)}{2}\right)\right] .
\end{array}\right.
\end{aligned}
$$

Note: If $w=\left(\frac{1}{n}, \frac{1}{n}, \cdots, \frac{1}{n}\right)$, then formula (28) is reduced to formula (6).

\section{Application to Multi-criteria Decision Making}

In many real-life cases, decision makers usually cannot choose but provide their preferences in the form of fuzzy information as a result of vague knowledge about the preference of alternatives. Therefore, a lot of fuzzy multicriteria decision making approaches have been developed and applied to diverse fields, such as engineering, management, economics, and so on. In this section, we present a method to solve multi-criteria decision making problems with the help of weighted fuzzy inaccuracy measure proposed by us.

Let $M=\left\{M_{1}, M_{2}, \ldots, M_{m}\right\}$ be a set of options, $C=\left\{C_{1}, C_{2}, \ldots, C_{n}\right\}$ be a set of criteria with weight vector $w=\left(w_{1}, w_{2}, \ldots, w_{n}\right)^{T}$, where $w_{j} \geq 0,(j=1,2, \ldots, n)$ and $\sum_{j=1}^{n} w_{i}=1$. The characteristics of the option $M_{i}$ in terms of criteria $C$ are represented by the following FSs:

$$
M_{i}=\left\{\left\langle C_{j}, \mu_{i j}\right\rangle \mid C_{j} \in C\right\}, i=1,2, \ldots, m
$$

and $j=1,2, \ldots, n$,

where $\mu_{i j}$ indicates the degree that the option $M_{i}$ satisfies the criterion $C_{j}$.

Using the measure defined by (28), we introduce the following approach to solve the above multi-criteria fuzzy decision making problem:

Step 1: Find out the positive-ideal solution $M^{+}$and negative-ideal solution $M^{-}$:

$$
\begin{aligned}
& M^{+}=\left\{\left\langle\mu_{1+}\right\rangle,\left\langle\mu_{2+}\right\rangle, \ldots,\left\langle\mu_{n+}\right\rangle\right\}, \\
& M^{-}=\left\{\left\langle\mu_{1-}\right\rangle,\left\langle\mu_{2-}\right\rangle, \ldots,\left\langle\mu_{n-}\right\rangle\right\},
\end{aligned}
$$

where, for each $j=1,2, \ldots, n$,

$$
\left.\begin{array}{l}
\left\langle\mu_{j+}\right\rangle=\left\langle\max _{i} \mu_{i j}\right\rangle \\
\left\langle\mu_{j-}\right\rangle=\left\langle\min _{i} \mu_{i j}\right\rangle
\end{array}\right\} .
$$

Step 2: Calculate $K\left(M^{+} ; M_{i}, w\right)$ and $K\left(M^{-} ; M_{i}, w\right)$ given respectively by the following:

$$
\begin{aligned}
& K\left(M^{+} ; M_{i}, w\right) \\
& =-\sum_{j=1}^{n} w_{j}\left[\begin{array}{r}
\mu_{j+}\left(x_{i}\right) \log \left(\frac{\mu_{j+}+\mu_{i j}}{2}\right) \\
\left.+\left(1-\mu_{j+}\right) \log \left(\frac{2-\mu_{j+}-\mu_{i j}}{2}\right)\right],
\end{array}\right.
\end{aligned}
$$


And

$$
\begin{aligned}
& K\left(M_{i} ; M^{-}, w\right) \\
& =-\sum_{j=1}^{n} w_{j}\left[\begin{array}{r}
\mu_{j-}\left(x_{i}\right) \log \left(\frac{\mu_{j-}+\mu_{i j}}{2}\right) \\
+\left(1-\mu_{j-}\right) \log \left(\frac{2-\mu_{j-}-\mu_{i j}}{2}\right)
\end{array}\right] .
\end{aligned}
$$

Step 3: Calculate the relative weighted fuzzy inaccuracy measure $K\left(M_{i}, w\right)$ of option $M_{i}$ with respect to $M^{+}$and $M^{-}$, where

$$
\begin{aligned}
K\left(M_{i}, w\right)= & \frac{K\left(M^{+} ; M_{i}, w\right)}{K\left(M^{+} ; M_{i}, w\right)+K\left(M^{-} ; M_{i}, w\right)}, \\
& i=1,2, \ldots, m
\end{aligned}
$$

Step 4: Select the option $M_{k}$ with s mallest $K\left(M_{k}, w\right)$.

\section{A Numerical Example}

In order to demonstrate the applicability of the proposed method to multi-criteria decision making, we consider below an investment company decision-making problem.

Example: Suppose that an investment company wants to invest a certain amount of money in the best option out of five options: A car company $M_{1}$, a food company $M_{2}$, a computer company $M_{3}$, an arms company $M_{4}$ and a TV company $M_{5}$. The investment company needs to take a decision according to the following four criteria: (1) $G_{1}$ is the risk analysis (2) $G_{2}$ is the growth analysis (3) $G_{3}$ is the social-political impact analysis (4) $G_{4}$ is the environmental impact analysis. Let $w=(0.20,0.18,0.32,0.30)^{T}$ be the criteria weight vector. The five possible options $M_{i}(i=1,2, \ldots, m)$ are to be evaluated by the decision maker under the above four criteria in the following form:

$$
\begin{aligned}
& M_{1}=\left\{\left\langle G_{1}, 0.5\right\rangle,\left\langle G_{2}, 0.6\right\rangle,\left\langle G_{3}, 0.3\right\rangle,\left\langle G_{4}, 0.2\right\rangle\right\}, \\
& M_{2}=\left\{\left\langle G_{1}, 0.7\right\rangle,\left\langle G_{2}, 0.7\right\rangle,\left\langle G_{3}, 0.7\right\rangle,\left\langle G_{4}, 0.4\right\rangle\right\}, \\
& M_{3}=\left\{\left\langle G_{1}, 0.6\right\rangle,\left\langle G_{2}, 0.5\right\rangle,\left\langle G_{3}, 0.5\right\rangle,\left\langle G_{4}, 0.6\right\rangle\right\}, \\
& M_{4}=\left\{\left\langle G_{1}, 0.8\right\rangle,\left\langle G_{2}, 0.6\right\rangle,\left\langle G_{3}, 0.3\right\rangle,\left\langle G_{4}, 0.2\right\rangle\right\}, \\
& M_{5}=\left\{\left\langle G_{1}, 0.6\right\rangle,\left\langle G_{2}, 0.4\right\rangle,\left\langle G_{3}, 0.7\right\rangle,\left\langle G_{4}, 0.5\right\rangle\right\} .
\end{aligned}
$$

By step 1, we obtain the positive-ideal solution $M^{+}$ and the negative ideal solution $M^{-}$:

$$
M^{+}=\left\{\left\langle G_{1}, 0.8\right\rangle,\left\langle G_{2}, 0.7\right\rangle,\left\langle G_{3}, 0.7\right\rangle,\left\langle G_{4}, 0.6\right\rangle\right\},
$$

$$
M^{-}=\left\{\left\langle G_{1}, 0.5\right\rangle,\left\langle G_{2}, 0.4\right\rangle,\left\langle G_{3}, 0.3\right\rangle,\left\langle G_{4}, 0.2\right\rangle\right\} .
$$

By step 2, we use formulas (30) and (31) to measure $K\left(M^{+} ; M_{i}, w\right)$ and $K\left(M^{-} ; M_{i}, w\right)$, respectively, we get the following tables:

Table 1: Values of $K\left(M^{+} ; M_{i}, w\right)$

\begin{tabular}{|c|c|}
\hline$K\left(M^{+} ; M_{1}, w\right)$ & 0.8662 \\
\hline$K\left(M^{+} ; M_{2}, w\right)$ & 0.7457 \\
\hline$K\left(M^{+} ; M_{3}, w\right)$ & 0.7685 \\
\hline$K\left(M^{+} ; M_{4}, w\right)$ & 0.8407 \\
\hline$K\left(M^{+} ; M_{5}, w\right)$ & 0.7642 \\
\hline
\end{tabular}

Table 2: Values of $K\left(M^{-} ; M_{i}, w\right)$

\begin{tabular}{|c|c|}
\hline$K\left(M^{-} ; M_{1}, w\right)$ & 0.7557 \\
\hline$K\left(M^{-} ; M_{2}, w\right)$ & 0.7547 \\
\hline$K\left(M^{-} ; M_{3}, w\right)$ & 0.7569 \\
\hline$K\left(M^{-} ; M_{4}, w\right)$ & 0.7535 \\
\hline$K\left(M^{-} ; M_{5}, w\right)$ & 0.7620 \\
\hline
\end{tabular}

By step 3, we obtain the relative weighted fuzzy inaccuracy measure $K\left(M_{i}, w\right)$ of option $M_{i}$ with respect to $M^{+}$and $M^{-}$by using the formula (32). We get the following table:

Table 3: Values of $K\left(M_{i}, w\right)$

\begin{tabular}{|c|c|}
\hline$K\left(M_{1}, w\right)$ & 0.5341 \\
\hline$K\left(M_{2}, w\right)$ & 0.4970 \\
\hline$K\left(M_{3}, w\right)$ & 0.5038 \\
\hline$K\left(M_{4}, w\right)$ & 0.5273 \\
\hline$K\left(M_{5}, w\right)$ & 0.5007 \\
\hline
\end{tabular}

Table 3 shows that the ranking order of five options is:

$$
M_{2} \succ M_{5} \succ M_{3} \succ M_{4} \succ M_{1} \text {. }
$$

Thus $M_{2}$ is the most desirable option.

\section{Conclusions}

In this work we have proposed a new fuzzy inaccuracy measure to measure the inaccuracy of a fuzzy set with respect to another fuzzy set. This measure is a 
modified version of fuzzy inaccuracy proposed by Verma and Sharma [11]. Some properties of the new fuzzy inaccuracy measure are investigated in detail. Furthermore, based on the weighted fuzzy inaccuracy, an approach to deal with multi-criteria decision-making problems under fuzzy environments is developed. Finally, an example is provided to illustrate the multicriteria decision-making process.

\section{References}

[1] L.A. Zadeh, Fuzzy sets, Information and Control, vol. 8, no. 3, 1965, 338-353.

[2] L.A. Zadeh, Probability measure of fuzzy event, Journal of Mathe matical Analysis and Applications, vol. 23, no. 2, 1968, 421-427.

[3] C.E. Shannon, A mathematical theory of communication, Bell System Technical Journal, vol. 27, 1948, 379-423; 623-656.

[4] A. De Luca and S. Termini, A definition of nonprobabilistic entropy in the setting of fuzzy set theory, Information and Control, vol. 20, no. 4, 1972, 301-312.

[5] D. Bhandari and N.R. Pal, Some new information measure for fuzzy sets, Information Science, vol. 67, no. 3, 1993, 209-228.

[6] S. Kullback and R.A. Leibler, On information and sufficiency, The Annals Mathematical Statistics, vol. 22, no. 1, 1951, 79-86.

[7] S. Kullback, Information Theory and Statistics, Dover Publication, 1968.

[8] J. Lin, Divergence measure based on Shannon entropy, IEEE Transaction on Information Theory, vol. 37, no. 1, 1991, 145-151.

[9] X. Shang and G. Jiang, A note on fuzzy information measures, Pattern Recognition Letters, vol. 18 , no. 5 , 1997, pp. 425-432.

[10] D. Kerridge, Inaccuracy and inference, Journal of Royal Statistical Society B, vol. 23, no. 1, 1961, 184-194.

[11] R.K. Verma and B.D. Sharma, A measure of inaccuracy between two fuzzy sets, Cybernetics and Information Technologies, vol. 11, no. 2, 2011, 13-23.

\section{Authors' Profiles}

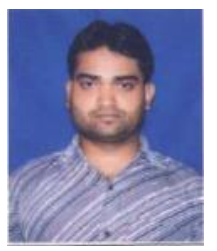

Rajkumar Verma is a Ph.D. student in Department of Mathematics, Jaypee Institute of Information Technology (Deemed University), Noida, (INDIA). $\mathrm{He}$ received the B.Sc. and M.Sc. degrees from Chaudhary Charan Singh
University (formerly, Meerut University), Meerut in 2004 and 2006 respectively. His research interests include Information Theory, Fuzzy information measures, Pattern recognition, Multicriteria Decisionmaking analysis, Soft set theory.

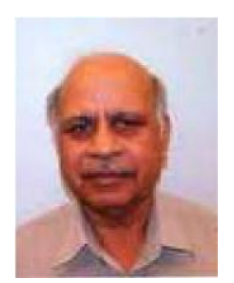

Bhu Dev Sharma currently Professor of Mathematics at JIIT-University, Noida, India did his Ph.D. from University of Delhi. He was a faculty at the University of Delhi (1966-1979) and earlier at other places. He spent about 29 years abroad 20 years (19882007) in USA and 9 years (1979-1988) in the West Indies, as Professor of Mathematics and in between as chair of the department.

Professor Sharma has published over 130 research papers in the areas covering Coding Theory, Information Theory, Functional Equations, Fuzzy Information, Intuitionistic Fuzzy Set Theory, Aggregation Operators and Statistics. He has successfully guided 24 persons for Ph.D. in India and abroad. He has also authored/coauthored 23 published books in Mathematics and some others on Indian studies. He is the Chief Editor of Jr. of Combinatorics, Information and System Sciences-JCISS (1976-present), and is on the editorial boards of several other journals.

$\mathrm{He}$ is member of several professional academic organizations: Past President of Forum for Interdisciplinary Mathematics (FIM), Vice President of Calcutta Mathematical Society, Ramanujan Math Society, Vice President of Academy of Discrete Mathematics and Applications (ADMA). After returning to India, he is working on establishing a Center of Excellence in Interdisciplinary Mathematics (CEIM) in Delhi, India.

Professor Sharma has received several awards and recognitions in India and abroad. He is widely traveled that would include Germany and several other countries of Europe, Brazil, Russia, China, and Japan. He has organized several International Conferences on Math/Stat in India, USA, Europe, Australia, China and Japan. Currently four persons are registered for Ph.D. under his supervision.

How to cite this paper: Rajkumar Verma, Bhu Dev Sharma,"A New Inaccuracy Measure for Fuzzy Sets and its Application in Multi-Criteria Decision Making", International Journal of Intelligent Systems and Applications(IJISA), vol.6, no.5, pp.62-69, 2014. DOI: 10.5815/ijisa.2014.05.06 